\title{
Rotational Remapping in Human Spatial Memory during Eye and Head Motion
}

\author{
W. Pieter Medendorp, ${ }^{1,2,3}$ Michael A. Smith, ${ }^{1,2,3}$ Douglas B. Tweed, ${ }^{1,2,4}$ and J. Douglas Crawford ${ }^{1,2,3}$ \\ ${ }^{1}$ Canadian Institutes of Health Research Group for Action and Perception, ${ }^{2}$ Centre for Vision Research, and ${ }^{3}$ Department \\ of Psychology, York University, Toronto, Ontario, Canada M3J 1P3, and ${ }^{4}$ Departments of Physiology and Medicine, \\ University of Toronto, Toronto, Ontario, Canada M5S 1 A8
}

The brain uses vision and other senses to compute the locations of objects relative to the body, and then must update these locations when the body moves. How geometrically sophisticated is this internal updating? It has been suggested that updating simply shifts the stored locations of all objects uniformly, by a common vector, when the eye or head turns. For horizontal and vertical turns, a uniform shift would often approximate the real changes in location of objects in front of the subject. But for torsional rotations, a shift would be inadequate: accurate updating would call for a more geometrically exact

From its sensory inputs, the brain computes the locations of surrounding objects. How is this geometric information handled? A central problem is that vision reports the locations of objects relative to the retina $\left(10^{\circ}\right.$ right of the fovea, for instance, or $5^{\circ}$ below), but these coordinates become obsolete as soon as the eyes move. Nevertheless the brain manages to keep track of objects in the dark, using remembered visual information, despite motion of the eyes or head. Humans and monkeys can look accurately to the remembered location of a flashed light, even when another eye or head movement intervenes between the target flash and the look (Hallet and Lightstone, 1976; Sparks and Mays, 1983; McKenzie and Lisberger, 1986; Israel and Berthoz, 1989; Pelisson et al., 1989; Schlag et al., 1990; Ohtsuka, 1994; Zivotofsky et al., 1996; Blouin et al., 1998; Herter and Guitton, 1998).

How could this space constancy be realized? Two mechanisms have been proposed. The brain may convert visual information from its original, retinal frame to a more stable frame, computing and storing the locations of objects relative to the Earth (or torso or inertial frame), so that the stored coordinates remain correct when the eyes and head move (Mays and Sparks, 1980; Andersen et al., 1985; Soechting et al., 1991). Alternatively, the brain may store locations of objects in a retinal frame (Goldberg and Bruce, 1990; Duhamel et al., 1992a; Walker et al., 1995). In that case, it would have to recompute all those locations every time the eyes moved, but there would be compensating advantages (Henriques et al., 1998). For either mechanism, visual spatial information

Received Sept. 17, 2001; revised Oct. 17, 2001; accepted Oct. 18, 2001.

This work was supported by grants from the Canadian Natural Sciences and Engineering Research Council. W.P.M. is supported by the Human Frontier Science Program. M.A.S. holds an Ontario Graduate Scholarship. D.B.T. is a Canadian Institutes of Health Research Scientist. J.D.C. is supported by the Canadian Research Chair Program. We thank J. Martinez-Trujillo for comments on this manuscript.

Correspondence should be addressed to Dr. W. P. Medendorp, Department of Psychology, York University, 4700 Keele Street, Toronto, Ontario, Canada M3J 1P3. E-mail: pieter@yorku.ca.

Copyright (ㄷ) 2001 Society for Neuroscience $\quad 0270-6474 / 01 / 220001-04 \$ 15.00 / 0$ remapping, not shifting but rotating the stored locations through the inverse of the rotation of the eye in space. Here we asked human subjects to make eye saccades to remembered targets after torsional head rotations. Their accuracy showed that spatial updating works in the torsional dimension and operates by rotation rather than shifting.

Key words: remapping; coordinates; spatial perception; eyehead movements; three-dimensional; human; spatial memory; rotation; rotational geometry

must be remapped. Eye-centered locations must be continuously updated or they must be converted to a more stable frame and later converted again, to limb-, eye-, or head-related coordinates for motor planning.

How geometrically sophisticated is this remapping? It has been suggested that updating might work with simple, geometrically crude operations (e.g., shifting the stored locations of all objects uniformly, by a common vector, when the eye turns) (Goldberg and Bruce, 1990; Duhamel et al., 1992b; Quaia et al., 1998). But a uniform shift merely approximates the geometrically exact remapping, which would involve rotating the stored locations through the inverse of the rotation of the eye in space, turning them all $30^{\circ}$ left when the eye turns $30^{\circ}$ right (Henriques et al., 1998; Smith and Crawford, 2001). Can spatial memory perform operations of this kinematic complexity?

Here we test whether visual memory can be rotationally remapped. The clearest test involves torsional eye and head motion (clockwise or counterclockwise, or in other words right or left ear down), because in this case the exact remapping differs markedly from a uniform shift. When the eye turns clockwise, then the world turns counterclockwise on the retina. Objects above the fovea move leftward in the retina-fixed frame, and objects below move rightward; objects right and left of the fovea

This article is published in The Journal of Neuroscience, Rapid Communications Section, which publishes brief, peerreviewed papers online, not in print. Rapid Communications are posted online approximately one month earlier than they would appear if printed. They are listed in the Table of Contents of the next open issue of JNeurosci. Cite this article as: JNeurosci, 2002, 22:RC196 (1-4). The publication date is the date of posting online at www.jneurosci.org.

http://www.jneurosci.org/cgi/content/full/5962 

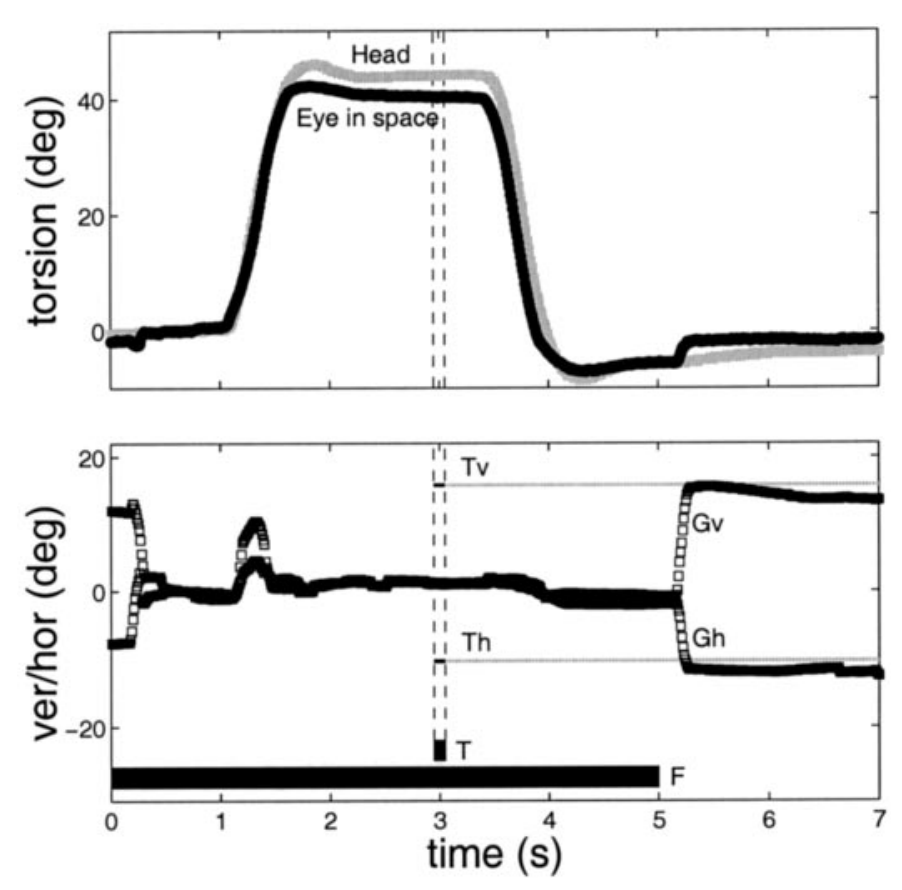

Figure 1. A typical trial illustrating the experimental paradigm. Top, The torsional orientation (in degrees) of the eye in space (black) and the head ( gray). Bottom, The pointing direction of the eye $(G v, G h)$; thin gray lines $(T v, T h)$ indicate the direction of the target. Thick black boxes mark the durations of the fixation target $(F)$ and the flashed target $(T)$. See Materials and Methods for explanation. The vertical dashed lines indicate the time interval during which the target was presented.

move up and down; so no uniform shift can match even approximately the true, rotary transformation. Surprisingly, however, spatial updating has never been studied in the torsional dimension. Here we asked human subjects to make eye saccades to remembered targets after torsional eye and head rotations.

\section{MATERIALS AND METHODS}

All experiments were approved by the Human Participants Review Subcommittee of York University. Our subjects were six healthy volunteers aged 21-31. They were seated and restrained to minimize trunk motion. The left eye was patched. We measured the three-dimensional (3-D) orientations of the right eye and the head using search coils (Skalar, Delft, The Netherlands) in three mutually orthogonal magnetic fields generated by field coils $2 \mathrm{~m}$ across. The three voltages from each coil were sampled at $100 \mathrm{~Hz}$ and converted into 3 -D eye and head positions, expressed as quaternions, and two-dimensional pointing directions (Tweed et al., 1990). Calibration and accuracy were as described previously (Henriques et al., 1998; Klier and Crawford, 1998).

Subjects faced a black tangent screen $2 \mathrm{~m}$ away in complete darkness. Green light-emitting diodes $\left(2 \mathrm{~cd} / \mathrm{m}^{2}\right)$ mounted on this screen served as targets. The target array consisted of 29 diodes: a central fixation light directly in front of the subject, 4 cardinal targets $\left(20^{\circ}\right.$ right, left, above, and below center), and 24 other targets distributed evenly among the four quadrants (six in each) at random locations between $15^{\circ}$ and $25^{\circ}$ eccentricity.

Experimental paradigm. In each trial, the subject began by fixating the illuminated central target $(F)$ while looking straight ahead (Fig. 1). Subjects were then instructed to rotate the head to one of five torsional orientations. These orientations were indicated by verbal commands, using clock-face numbers $10,11,12,1$, and $2 ; 10$ o'clock meant maximum counterclockwise head torsion $\left(\sim 45^{\circ}\right), 12$ o'clock meant upright (i.e., no head rotation was required), and 2 o'clock meant maximum clockwise torsion. While the subject maintained this head position, $\sim 3 \mathrm{sec}$ after the start of the trial, one of the peripheral targets $(T)$ flashed for $100 \mathrm{msec}$. Then the subject straightened his or her head to the upright 12 o'clock position while still fixating the central light. Approximately $2 \mathrm{sec}$ later, the central light switched off and an audio tone cued the subject to look

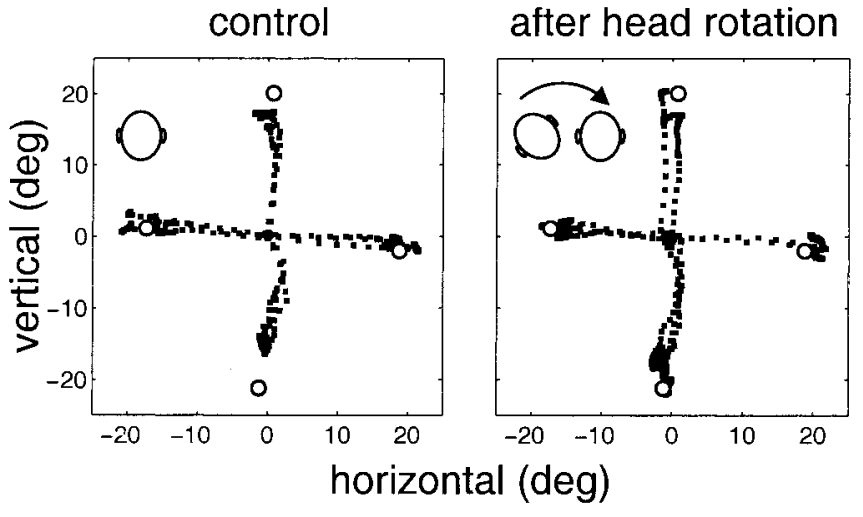

Figure 2. One subject's performance in the control condition (left) and in the "after head-tilt" condition (right). Saccades to the four cardinal targets (○) are shown. In the control condition the subject made no head movements before saccading to the remembered target. In the after head-tilt condition, the subject perceived the target at a $45^{\circ}$ rightward head tilt, rolled his head back to upright, and subsequently made the saccade. In both conditions, saccadic directions were accurate.

to the remembered location of the flashed target, keeping the head still. [When the head was tilted, the eyes counter-rolled slightly, $\sim 4.7^{\circ}$ on average for $45^{\circ}$ of head torsion, as in other studies (Haslwanter et al., 1992). By having subjects hold the head upright during their gaze shifts, we eliminated any effect of counter-roll on their saccades (Klier et al., 1998).] The entire trial lasted $\sim 7 \mathrm{sec}$. Targets and head orientations were selected randomly. For each of the five head orientations, the four cardinal targets were tested three times each and the other 24 targets were tested once each, for a total of 180 trials. Note that the 12 o'clock target enabled us to obtain the subject's memory-guided saccades in the absence of updating. Typically eight trials per subject were excluded from additional analysis because the subject failed to follow the above instructions.

In a control test, the subject was seated with his or her head upright and saccaded from the central target to each of the peripheral targets, which were illuminated for $1.5 \mathrm{sec}$. In this way, we obtained the ideal gaze directions for each of the targets.

\section{RESULTS}

Figure 2 shows the gaze trajectories of subject S.M. toward the four remembered cardinal targets (Fig. 2, ○) for the control condition (i.e., without head rotation; Fig. 2, left ) and after a head tilt of $\sim 45^{\circ}$ (Fig. 2, right). In the control condition, this subject often undershot targets, but his saccade directions were accurate. In the test condition (Fig. 2, right), with an intervening head rotation, the subject viewed the flashed targets from a $45^{\circ}$ clockwise head tilt before righting the head and finally performing the saccades. As the figure shows, saccades were accurate for all four targets, indicating almost perfect compensation for the head and eye rotation.

What would have happened in the test condition if the subject's compensation had been imperfect? When the head is tilted $45^{\circ}$ clockwise, the eye is tilted $\sim 40^{\circ}$ clockwise relative to space, so if the subject had not corrected at all for the intervening eye rotation in space (i.e., if there had been no remapping) his saccades would have been misdirected by $\sim 40^{\circ}$ counterclockwise. (For example, if the head is tilted $45^{\circ}$ clockwise, and the eye is tilted $40^{\circ}$ clockwise in space, and a target appears in the 12 o'clock position on a space-fixed clock face, then the retina will see the target on a meridian tilted $40^{\circ}$ counterclockwise. If the subject rights his head and then makes a saccade along that retinal meridian, his directional error will be $40^{\circ}$.) If the subject had corrected for the rotation of the eye in the head, rather than its rotation in space, he would have been off by $45^{\circ}$ counterclock- 


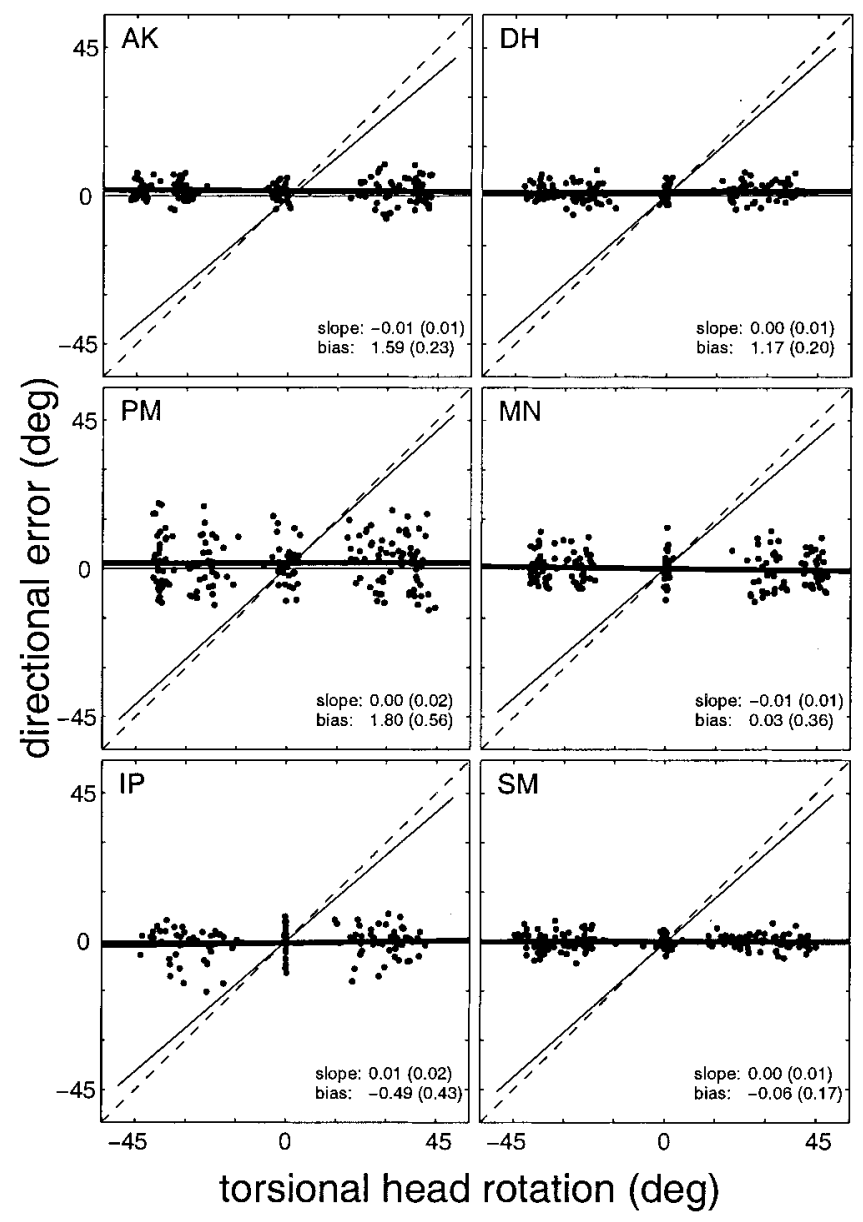

Figure 3. Directional errors of all subjects plotted against the size of the intervening head rotation (black dots). Subjects varied in their accuracy, but none showed a systematic relationship between direction error and head rotation: regression lines (thick lines) had slopes near zero. The slopes would have equaled 0.9 (thin solid line) if subjects had not corrected for the torsion of the eye in space (because of a small ocular counter-roll, eye torsion in space is $90 \%$ of head torsion in space). The dashed lines indicate updating for eye in head torsion but not for head torsion in space.

wise. If he had corrected for the rotation of the head in space rather than the rotation of the eye, he would have misaimed by $\sim 5^{\circ}$ clockwise.

Figure 3 shows the data of all our subjects, plotting directional error versus the intervening head rotation. Because eye responses often consisted of several saccades, we measured the directional error of the first saccade toward the remembered target. Subjects differed in their average error, varying between $2.2^{\circ}$ and $7.5^{\circ}$. But across all subjects, the data scattered around zero, showing no systematic directional errors.

Using linear regression, we quantified the relationship between directional error and head rotation. If there had been no updating for the torsion of the eye in space, the line would have had a slope of $\sim 0.9$. If the updating had corrected for the torsion of the eye in the head but not the torsion of the head in space, the slope would have been 1.0; if it had corrected for the torsion of the head but not the torsion of the eye, the slope would have been -0.1 . Perfect updating would yield a slope of 0 . The results favor the last hypothesis: across all subjects the slopes ranged between -0.02 and 0.02 , indicating almost ideal performance. On average, the slope was not significantly different from $0(t$ test; $p=0.9)$ but

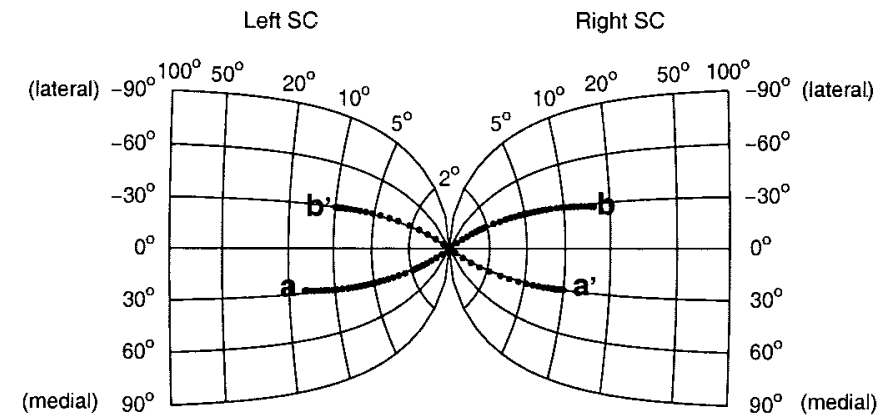

Figure 4. Rotational remapping in the collicular map. A space-fixed target $a\left(10^{\circ}\right.$ leftward and $30^{\circ}$ upward $)$ that is flashed when the eye is turned $45^{\circ}$ counterclockwise stimulates the left side of the retina. Therefore it is represented on the left SC. But after the eye rotates upright, the remembered target is now to the right relative to the retina. In the collicular map, then, its representation must cross the midline, from the left to the right SC (see $\left.a^{\prime}\right)$. At the same time, target $b\left(10^{\circ}\right.$ rightward and $30^{\circ}$ downward in space) should cross in the opposite direction, from the right $\mathrm{SC}$ to the left $\left(\right.$ see $\left.b^{\prime}\right)$.

was significantly different from -0.1 ( $t$ test; $p<0.001)$ and from 0.9 and 1 ( $t$ test; $p<0.001$ ).

Could our subjects have achieved this accuracy by shifting, rather than rotating, the stored locations of the targets? Geometrically, the shift that best approximates a torsional rotation is the null shift (no shift at all), because any other uniform motion would improve the spatial information on one side of the fovea but make it less accurate on the other side. So at best, updating by shifting would lead to errors like those shown in Figure 3 for the no-remapping strategy, with its slope of 0.9. Figure 3 shows, then, that our subjects were far more accurate than is consistent with updating by a uniform shift. Their updating closely approximated the optimal, rotational remapping.

\section{DISCUSSION}

Our subjects made almost no systematic directional errors when looking to remembered targets after intervening torsional eye and head movements. It has already been shown that spatial memory copes well with horizontal and vertical eye and head motion. Our results show that it also corrects for torsional motion. Because these experiments were performed in total darkness, the system must have relied solely on extra-retinal signals. Together with previous results, we can now say that these signals account for all three dimensions of eye and head rotation: horizontal, vertical, and torsional. Here we consider the physiological and computational implications of this finding.

Some authors have suggested that remapping might operate by very simple geometry, merely shifting all stored locations by the same, common vector whenever the eyes turn (Goldberg and Bruce, 1990; Moschovakis and Highstein, 1994). This uniform shift would approximate fairly well the real changes of location during most horizontal and vertical eye movements, but it would cause a pattern of cumulative inaccuracy for eye movements between eccentric targets. When tested on this task, subjects do not show the predicted errors (Smith and Crawford, 2001). Their accuracy suggests that updating is more sophisticated than a simple shift, that it more closely approximates the geometrically exact remapping, rotating stored locations through the inverse of the rotation of the eye. Smith and Crawford (2001) studied horizontal and vertical saccades with the head fixed. In the present study, we have tested their scheme in a far more dramatic 
and previously untested situation. We show accurate, rotational remapping in a more computationally demanding task, during eye and head motions in the torsional dimension. Together with the results obtained by Smith and Crawford (2001), our findings suggest that spatial updating operates by rotational remapping in all three dimensions (horizontal, vertical, and torsional) and accurately combines information about the movements of the eyes and head. Remapping may work with efference copy or vestibular inputs (Blouin et al., 1998). It may operate entirely in retinal coordinates, it may use other head- or Earth-fixed coordinates, or it may involve mixed or distributed representations (Pouget and Sejnowski, 1997). But our results show that the net result is an accurate, rotational remapping of object locations.

Our data suggest new experimental predictions for the neural structures that participate in remapping, such as the posterior parietal cortex, the frontal cortex, and the superior colliculus (SC) (Goldberg and Bruce, 1990; Duhamel et al., 1992a; Walker et al., 1995). Figure 4 shows the predicted neurophysiological consequences of torsional remapping in the SC (Munoz et al., 1991; Klier et al., 2001). A space-fixed target $a$, flashed when the eye is turned $45^{\circ}$ counterclockwise, stimulates the left side of the retina. Therefore, it is represented on the left SC. But after the eye rotates upright, the remembered target is now on the right relative to the retina. In the collicular map, then, its representation must cross the midline, from the left to the right SC (Munoz et al., 1991; Walker et al., 1995; Pouget and Snyder, 2000). At the same time, target $b$ should cross in the opposite direction, from the right side of the retina to the left, and therefore from the right SC to the left. That is, a torsional motion of the eye or head should rotate the activity pattern on the collicular map, moving different active sites in opposite directions across the midline of the brain.

\section{REFERENCES}

Andersen RA, Essick GK, Siegel RM (1985) Encoding of spatial location by posterior parietal neurons. Science 230:456-458.

Blouin J, Labrousse L, Simoneau M, Vercher JL, Gauthier GM (1998) Updating visual space during passive and voluntary head-in-space movements. Exp Brain Res 122:93-100.

Duhamel JR, Colby CL, Goldberg ME (1992a) The updating of the representation of visual space in parietal cortex by intended eye movements. Science 255:90-92.

Duhamel JR, Goldberg ME, Fitzgibbon EJ, Sirigu A, Grafman J (1992b) Saccadic dysmetria in a patient with a right frontoparietal lesion. The importance of corollary discharge for accurate spatial behaviour. Brain 115:1387-1402.

Goldberg ME, Bruce CJ (1990) Primate frontal eye fields. III. Maintenance of a spatially accurate saccade signal. J Neurophysiol 64:489-508.

Hallet PE, Lightstone AD (1976) Saccadic eye movements to flashed targets. Vision Res 16:107-114.
Haslwanter T, Straumann D, Hess BJM, Henn V (1992) Static roll and pitch in the monkey: shift and rotation of Listing's plane. Vision Res 32:1341-1348.

Henriques DYP, Klier EM, Smith MA, Lowy D, Crawford JD (1998) Gaze-centered remapping of remembered visual space in an open-loop pointing task. J Neurosci 18:1583-1594.

Herter TM, Guitton D (1998) Human head-free gaze saccades to targets flashed before gaze-pursuit are spatially accurate. J Neurophysiol 80:2785-2789.

Israel I, Berthoz A (1989) Contribution of the otoliths to the calculation of linear displacement. J Neurophysiol 62:247-263.

Klier EM, Crawford JD (1998) The human oculomotor system accounts for 3-D eye orientation in the visual-motor transformation for saccades. J Neurophysiol 80:2274-2294.

Klier EM, Wang H, Crawford JD (2001) The superior colliculus encodes gaze commands in retinal coordinates. Nat Neurosci 4:627-632.

Mays LE, Sparks DL (1980) Saccades are spatially, not retinotopically coded. Science 208:1163-1164.

McKenzie A, Lisberger SG (1986) Properties of signals that determine the amplitude and direction of saccadic eye movements in monkeys. J Neurophysiol 56:196-207.

Moschovakis AK, Highstein SM (1994) The anatomy and physiology of primate neurons that control rapid eye movements. Annu Rev Neurosci 17:465-488.

Munoz DP, Pelisson D, Guitton D (1991) Movement of neural activity on the superior colliculus motor map during gaze shifts. Science 251:1358-1360.

Ohtsuka K (1994) Properties of memory-guided saccades toward targets flashed during smooth pursuit in human subjects. Invest Ophthalmol Vis Sci 35:509-514.

Pelisson D, Guitton D, Munoz DP (1989) Compensatory eye and head movements generated by the cat following stimulation-induced perturbations in gaze position. Exp Brain Res 78:654-658.

Pouget A, Sejnowski TJ (1997) Spatial transformations in the parietal cortex using basis functions. J Cogn Neurosci 9:222-237.

Pouget A, Snyder LH (2000) Computational approaches to sensorimotor transformations. Nat Neurosci [Suppl] 3:1192-1198.

Quaia C, Optican LM, Goldberg ME (1998) The maintenance of spatial accuracy by perisaccadic remapping of visual receptive fields. Neural Netw 11:1229-1240.

Schlag J, Schlag-Rey M, Dassonville P (1990) Saccades can be aimed at the spatial location of targets flashed during pursuit. J Neurophysiol 64:575-581

Smith MA, Crawford JD (2001) Implications of ocular kinematics for the internal updating of visual space. J Neurophysiol 86:2112-2117.

Soechting JF, Flanders M, Helms Tillery SI (1991) Transformation from head- to shoulder-centered representation of target direction in arm movements. J Cogn Neurosci 2:32-43.

Sparks DL, Mays LE (1983) Spatial localization of saccade targets. I. Compensation for stimulation-induced perturbations in eye position. J Neurophysiol 49:45-63.

Tweed D, Cadera W, Vilis T (1990) Computing three dimensional eye position quaternions and eye velocity from search coil signals. Vision Res 30:97-110.

Walker MF, Fitzgibbon J, Goldberg ME (1995) Neurons of the monkey superior colliculus predict the visual result of impeding saccadic eye movements. J Neurophysiol 73:1988-2003.

Zivotofsky AZ, Rottach KG, Averbuch-Heller L, Kori AA, Thomas CW, Dell'Osso LF, Leigh RJ (1996) Saccades to remembered targets: the effects of smooth pursuit and illusory stimulus motion. J Neurophysiol 76:3617-3632. 